\title{
The Effect of Wing Chun Day Punch Boxing Based on Embedded Microprocessor
}

\author{
Sile Wang, ${ }^{1,2}$ Xiaodan Chen $\left(D,{ }^{3}\right.$ Yun Chen, ${ }^{4}$ Dong Liang, ${ }^{5}$ and Sichao $\mathrm{Fu}^{6}$ \\ ${ }^{1}$ College of Physical Education, Anqing Normal University, Anqing 246000, Anhui, China \\ ${ }^{2}$ College of Graduate, Kyungnam University, Changwon 51767, Republic of Korea \\ ${ }^{3}$ Wushu School, Guangzhou Sport University, Guangzhou 510500, Guangdong, China \\ ${ }^{4}$ Binary University of Management and Entrepreneurship, Puchong 47100, Malaysia \\ ${ }^{5}$ Department of Health Science and Industry, Myongji University, Seoul 03675, Republic of Korea \\ ${ }^{6}$ School of Mechanical and Automotive Technology, Jilin Vocational College of Industry and Technology, Jilin 132013, Jilin, China \\ Correspondence should be addressed to Xiaodan Chen; 11155@gzsport.edu.cn
}

Received 13 December 2021; Revised 13 January 2022; Accepted 21 January 2022; Published 12 February 2022

Academic Editor: Haibin Lv

Copyright (c) 2022 Sile Wang et al. This is an open access article distributed under the Creative Commons Attribution License, which permits unrestricted use, distribution, and reproduction in any medium, provided the original work is properly cited.

\begin{abstract}
With the popularity of Wing Chun film and television themes in recent years, Wing Chun has gradually received more and more attention, but the impact of Japanese punching boxing is rarely involved. The purpose of this article is to study the impact of Wing Chun Day Punch Boxing based on embedded microprocessor, to understand the actual effect of Wing Chun Day Punch Boxing through the analysis of the beat effect, and to provide reference and help for the research of traditional Chinese martial arts. In this paper, experimental methods and mathematical statistics combined with embedded microprocessors are used to research and analyze the punching effect of Wing Chun word punching. And it calculates the speed, angle, angular velocity, angular acceleration, muscle discharge sequence, and other data of various parts of the body during the process of Wing Chun force release to reveal the hitting effect of Wing Chun day word punching. Five Wing Chun practitioners with different training times are selected for Wing Chun day punch training, using engineering dummies for experiments, relying on acceleration sensors, position sensors, and force sensors. Starting from the direction of dynamics and kinematics, it collects the effective data of the Japanese word punch, then analyzes the experimental results through the embedded microprocessor, and obtains the hitting effect of the Wing Chun Japanese word punch. Experiments show that, through the analysis of the force angle and characteristics of the Japanese punch, the subjects' elbow joint angle changes significantly when doing the Japanese punch. In the experiment on the force measurement engineering dummy, when the subjects punched, the measurement results of the vibration acceleration of the internal organs of different subjects were $p>0.01$, and there was no significant difference. However, in the case of elbow joint $150^{\circ}$ preparation, the midline punch is significantly larger than the punch directly in front of the shoulder, and the difference is statistically significant (significant level $p<0.01)$.
\end{abstract}

\section{Introduction}

Traditional martial arts have attracted the attention of sports culture experts and scholars for its characteristics of physical fitness, spiritual martial arts development, and traditional cultural connotation. In the 1980s, the excavation and classification of traditional martial arts were booming, and the results brought great value to academic research. The
Wushu Management Center of the State Sports General Administration put forward the idea of developing "big martial arts" in 2011. This opened the prelude to the traditional martial arts research project of this century and guided the future research direction. However, the research of traditional martial arts also faces inevitable difficulties. Traditional Chinese martial arts have many sects and various types of martial arts, such as south fist and north foot, east 
gun and west stick, the theory of "internal and external family," Shaolin's "Zen fist as one body," and Wudang's Taoist martial arts. Many issues involve a wide range of issues, and the general public does not know the secrets among them. Science aims at pursuing the "truth" hidden behind the appearance of things and is expected to unravel the mystery of martial arts. However, scientific researchers are also limited by conditions, and researchers have various "misunderstandings" about traditional martial arts, which will inevitably limit their exploration direction, and even completely unrealistic hypotheses are designed, and some conclusions are difficult to verify. Because of deliberately avoiding similar research and opening a new way to show the superficial prosperity of traditional martial arts research, there is no in-depth study of the characteristics and essence of traditional martial arts. It removes them and summarizes them as a "whole" and directly draws the conclusion that this adds a sense of mystery to the mystery of traditional martial arts.

Wing Chun is one of the famous traditional martial arts in China because of its unique style and strong practicability. A large group of martial arts enthusiasts at home and abroad are practicing Wing Chun, and there is a saying in China that "Tai Chi in the north, Wing Chun in the south." Wing Chun practitioners often appear in some of the world's top fighting competitions, and it is known as second only to Tai Chi, one of the most popular boxing styles in the world. In recent years, with the popularity of Wing Chun, people have paid more and more attention to the research of Wing Chun. For all Wing Chun fans, conquering Wing Chun is the unanimous goal of everyone. The purpose of this article is to analyze the biomechanical characteristics of typical Wing Chun movements, provide a scientific basis for formulating movement specifications, analyze the effects of Wing Chun Japanese punching boxing, and provide objective and powerful support for movement teaching. The data obtained by using modern advanced biological power equipment, whether it is a direct explanation of Wing Chun theory or the supplement and amendment of certain theories, is an important breakthrough and innovation in traditional martial arts research, and it is of great significance; therefore, this research is about the real hitting effect of Wing Chun-style punching boxing. Through the biomechanical analysis of the single action of Japanese punching boxing, and based on the science of this type of boxing, it explores its real punching effect and provides a theoretical reference for boxing education. This research can not only give people a deeper understanding of Wing Chun, but also can be used to explore the characteristics, mechanisms, and methods of various martial arts and gradually reveal the mysteries of martial arts.

Boxing is a strenuous exercise that requires aerobic and anaerobic metabolism in a short period of time, and boxing also requires the coordination of upper body muscles and lower body muscles. Lee investigated the energy expenditure (EE) of boxing unit exercises, such as straight punches, uppercuts, and uppercuts and examined the impact of boxing training on unit exercises. Ten inexperienced college students ( 5 males and 5 females; $28.2 \pm 2.1$ years old, $171.4 \pm 8.4 \mathrm{~cm}, 65.0 \pm 10.1 \mathrm{~kg}$ ) were selected to participate in a 6 -week boxing training program (3 times/week, 40-60 minutes/session), and it determined nine boxing unit actions and formed 3 combinations of these unit actions. The energy expenditure of each unit exercise and exercise combination was measured before the training plan, in the $3 \mathrm{rd}$ and 6 th weeks. In order to compare the impact of the training period on energy expenditure, a one-way analysis of variance with repeated measures was applied. Experiments show that boxing training consumes a lot of energy, and different boxing moves consume different energy, but his research lacks a detailed study of boxing effects [1]. Kasiri et al. propose a robust framework for automatic classification of boxers' punches. They used overhead depth images to alleviate the challenges associated with occlusion and developed powerful body part tracking for noisy time-of-flight sensors. Punch recognition is solved by a multiclass support vector machine (SVM) and a random forest classifier using a combination of features. Based on the prior knowledge of boxing, they proposed a hierarchical SVM classifier from coarse to fine. The framework has been applied to the boxing image sequence taken by the Australian Institute of Sport and 14 elite boxers, and the results prove the effectiveness of the action recognition method. The hierarchical SVM classifier improves the accuracy of $97.3 \%$ on the most advanced action recognition system recently, but their research has limitations [2]. Lee et al. pointed out that boxing is a high-intensity exercise that requires both aerobic and anaerobic metabolic capacity in the short term. Boxing also requires coordination between the upper and lower body muscles. They investigated the energy expenditure (EE) of boxing unit movements, such as straights and uppercuts, and examined the effect of boxing training on energy expenditure per unit movement [3]. Filipe and Lins use fault injection and heavy ion experiments to study the impact of register file errors on the reliability of modern embedded microprocessors. In addition, they also evaluated how different levels of compiler optimization modify the use of processor register files and the probability of failure. They chose six representative benchmarks, each of which uses three different levels of compiler optimization to compile. Detailed fault injection activities were carried out to measure the vulnerability factors of the register architecture of each code and configuration and to identify registers that are more likely to cause silent data corruption or single event function interruptions. In addition, they correlated the observed reliability changes with the register file utilization. Finally, they irradiated two selected benchmarks with heavy ions; these benchmarks were compiled with two optimization levels, and the experimental results were correlated with the fault injection analysis. However, the factors they considered in the research on the factors affecting the reliability of embedded microprocessors were not comprehensive [4]. Sato et al. showed the design and experimental results of a 
fast single-flux quantum (RSFQ) bit-serial microprocessor, which has a reduced embedded random access memory (RAM) and a minimum instruction set, called CORE e2h. A microprocessor called the CORE e series has been developed to demonstrate the execution of small-scale programs such as cyclic calculations and sequencing to demonstrate the first prototype of a stored program computer using RSFQ technology. CORE e2h is the most simplified variant of the CORE e series, and it is only equipped with two registers and can execute 13 instructions. The target clock frequency for bit-serial operation is $50 \mathrm{GHz}$, and the designed system clock cycle is $2 \mathrm{GHz}$. Each component is carefully designed, using a small number of Josephson junctions to realize the function and occupying a small space. They manufactured multiple CORE e2h microprocessor chips and integrated two RAMs based on 128-bit shift registers on the same chip. They obtained the correct operation of all instructions through experiments, which was relatively stable. But they did not explain the performance of the microprocessor under the premise of maintaining stability [5]. Highly regular many-core architectures are becoming more and more popular, because they are suitable for inherently highly parallelized applications, such as most image and video processing fields. They proposed a novel architecture for many-core microprocessor ASICs dedicated to embedded video and image processing applications. They proposed a flexible multicore method with two architectures: one is implemented using CMOS $65 \mathrm{~nm}$ technology containing 16 open source blocks, and the other is implemented using CMOS FD-SOI $28 \mathrm{~nm}$ technology containing 64 open source blocks. Each block of these architectures can select its communication link based on the overall parallel scheme most relevant to the target application. Both chips have complete functions in the simulation, and the layout shows the results of frequency, area, and power consumption. They provide various case studies to illustrate the proposed flexible many-core architecture and can focus on architecture exploration, parallelized instantiation schemes, and timing performance. However, the architecture they proposed is too simple to give a detailed introduction to the architecture [6].

This article explains the striking effect of the Wing Chun Sun Punch Boxing from three aspects: the external performance and internal characteristics of the action. By testing the kinematics and dynamics parameters of Japanese punching boxing, combined with the in-depth analysis of Wing Chun boxing theory, it provides theoretical support for the standardization of movements and teaching programs. Through research and analysis of the changes in joint angles of Japanese punching movements and changes in EMG data, it compares the increased effect difference between midline punch and forward punch in Japanese punch. At the same time, it uses test engineering dummies for testing, and the engineering dummies are equipped with sensors that can cooperate with embedded processors to analyze the effect of Japanese punching, making the analysis results more accurate.

\section{The Effect of Wing Chun Japanese Punching Boxing Based on Embedded Sensors}

2.1. Wing Chun Day Punch Boxing. Traditional martial arts are divided into seven major boxing systems, each of which has its own system and its own characteristics. Wing Chun, as a branch of Nanquan of the seven major types of boxing, has the short-playing characteristics of Nanquan. At the same time, it pioneered the theory of "keeping the middle and using the middle, continuously eliminating and hitting, staying and sending, short bridges, avoiding the reality and eliminating the virtual, and eliminating without elimination" $[7,8]$. Figure 1 is a diagram of the simple moves of Wing Chun.

The characteristic of Japanese punch boxing is that the lower limbs basically do not move. When punching, there is a slight movement through the waist and hips, as shown in Figure 2. In kinematics, the angle of the lamb and horse is used to illustrate the stability of the lower limbs and maintain the balance of the body. It is beneficial to increase the hitting effect with the help of the changes in the angle of the body's joints and the ground reaction force $[9,10]$. The techniques and requirements of Japanese punching boxing: Japanese punching boxing is one of the basic attack methods of Wing Chun [11]. It requires two fists to be placed in front of the chest, the left palm is in front, the left arm is slightly bent, and the right palm is placed at the curve of the left elbow. Its fists face upwards, and the fist face resembles the character "day," so it is called the Japanese word punch. Fists clasped fists and underarms, forming a "two-character forceps Yangma," and when punching, the elbow is centered, the elbow is attached to the ribs, and the force of the elbow is used to punch in a straight line. Double fists are punched from the midline of the human body in turn, attacking the midline of the opponent's body, and retracted from the midline, that is, the principle of "keeping the center and using the center." This requires the neck, shoulders, elbows, wrists, waist, thighs, knees, and ankles to form a whole $[12,13]$. Pile work is extremely important when distributing inch strength: clamp the knee, levator anus, abdomen, hold the chest, pull the back, straighten the head, and ask in front of the hand; the whole body is shaped like an arrow, and with the help of the reaction force of the ground, the joints of the whole body are instantaneously energized in one direction, sending out the Cun Jin of Wing Chun [14].

2.2. Embedded Microprocessor. With the continuous advancement of science and technology, there is an increasing demand for equipment miniaturization and integration, and the entire processing system tends to be integrated into a smaller circuit board $[15,16]$. With the rapid development of current processor technology, its performance has doubled almost every year [17-19]. At the same time, the system requires high data processing speed and highly integrated processing chips to complete tasks that require multiple processing devices and peripheral devices [20, 21]. This requires the system to have a higher data processing speed and a highly integrated processing chip to complete the 

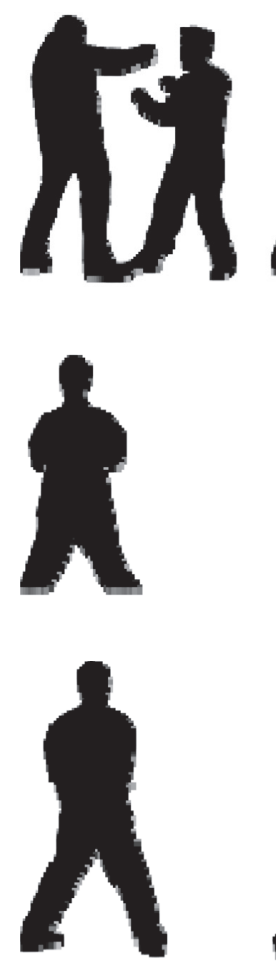
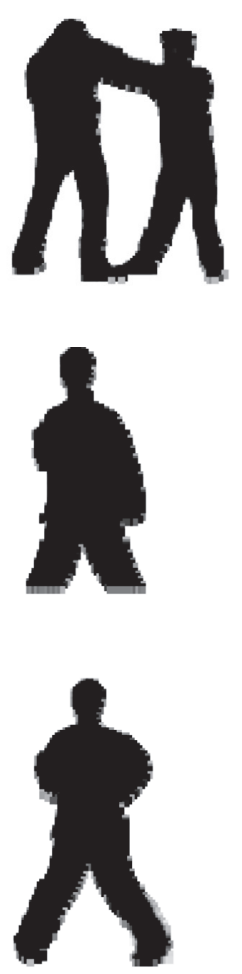

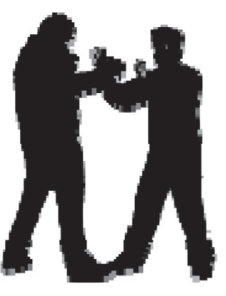

(a)

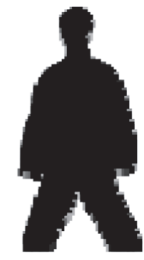

(b)

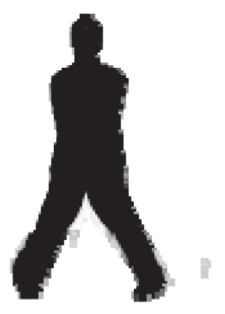

(c)
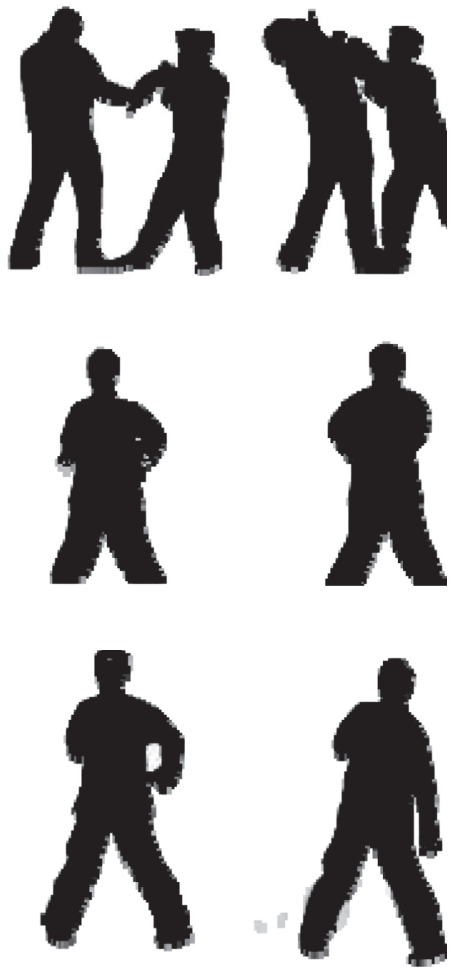

Figure 1: Simple strokes of Wing Chun.
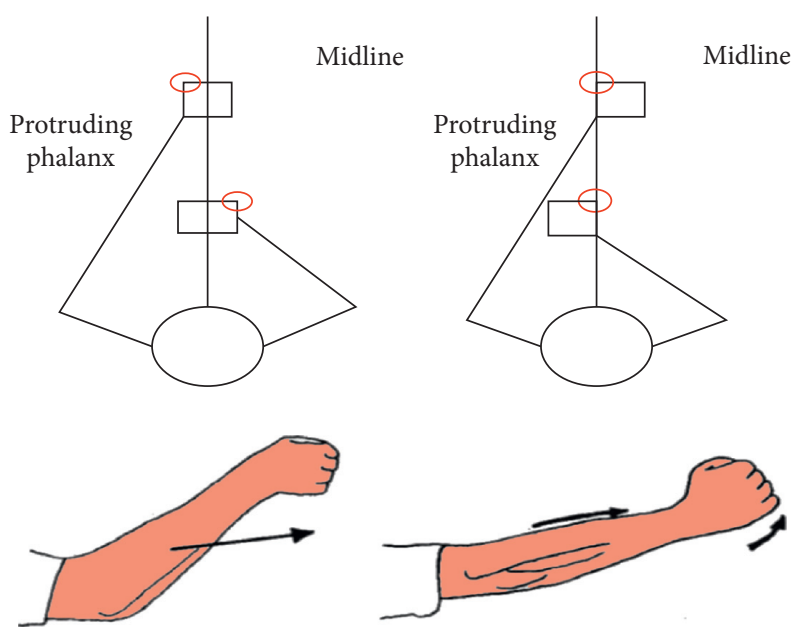

FIgURE 2: Illustration of Wing Chun day punch boxing.

original processing [22]. Embedded microprocessors can just meet this requirement, which has small size, rich system on chip, and can meet the design and production requirements only through the expansion of some simple external circuits [23]. At the same time, it has a wealth of interfaces, which can be provided to users for human-computer interaction, data communication, etc., and its price is becoming cheaper and cheaper, and it has a higher cost performance, which can effectively process and analyze data $[24,25]$.
The architecture of the embedded microprocessor is shown in Figure 3:

Through the research of embedded microprocessor, this paper chooses three kinds of sensors, force sensor, acceleration sensor, and position sensor, to measure and study the effect of Japanese punching. Based on the traditional impedance measurement method, the excitation voltage signal is applied to the series measurement circuit, the voltage of the standard resistance or the measurement resistance is monitored, and the relationship between the 


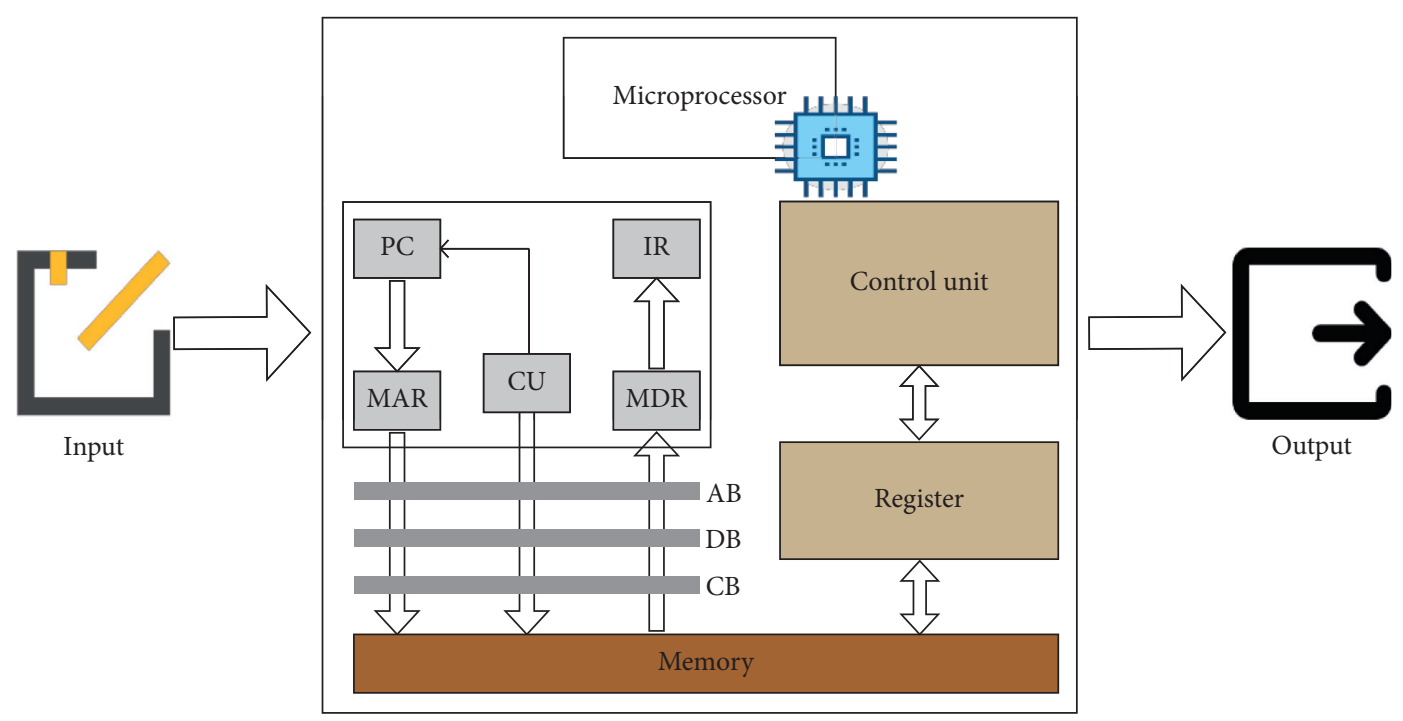

Figure 3: Embedded microprocessor architecture.

measured voltage and the excitation voltage is compared $[26,27]$. Finally, the measured resistance is calculated by comparing the amplitude ratio and phase difference between the excitation signal and the voltage across the standard resistance [28].

Based on the constant frequency characteristics that satisfy the input and output, the excitation and measurement signals can be assumed as follows:

$$
\left\{\begin{array}{l}
T_{a}(s)=\sin (\delta s) \\
T_{0}(s)=B \sin (\delta s+\varphi)
\end{array}\right.
$$

Among them, $T_{a}(s)$ is the excitation signal, $T_{0}(s)$ is the standard signal, and $B$ is the output signal amplitude.

At the same time, assuming that the standard resistance is $E$ and the excitation frequency is $\delta$, then it can be calculated:

$$
\left\{\begin{array}{l}
B=\frac{E}{\sqrt{(E+b)^{2}+a^{2}}} \\
\varphi=-b \tan \frac{a}{E+b}
\end{array}\right.
$$

The measurement solution $B$ is based on $\varphi$, and the real and imaginary parts $a, b$ of the measured impedance are obtained by inverse calculation by the following formula:

$$
\left\{\begin{array}{l}
B=(\varphi-1) E \\
A=(B+E) \tan \varphi
\end{array}\right.
$$

The real and imaginary parts $a$ and $b$ of the measured impedance are related to the excitation frequency, and in order to obtain the impedance of the measured object, the calculation can be obtained:

$$
\left\{\begin{array}{l}
\frac{T_{0}}{E_{1}}=\frac{T_{i}}{E_{1}}-b_{i}+b_{n} \\
\frac{T_{0}}{E_{2}}=b_{i}+b_{n}
\end{array}\right.
$$

Combining the formulas, we can deduce

$$
\frac{T_{0}-T_{1}}{E}=\frac{T_{i}}{E}-2 b_{i}+b_{n}
$$

This means that while eliminating the current noise, the voltage across the measured impedance is obtained, and the result is

$$
\begin{aligned}
& T_{d}=T_{0}-T_{1} \approx T_{i}-2 b_{i} E, \\
& T_{0}=2 b_{i} E=T_{i}-T_{d} .
\end{aligned}
$$

The output signal on the measured impedance can be calculated by the voltage difference calculation on the symmetrical resistor, thereby eliminating the interference of indoor current noise.

$$
I=\frac{T_{3}-T_{4}}{E_{3}+E_{4}+E_{i 1}+E_{5}}=\frac{T_{1}-T_{2}}{D_{x}},
$$

$T_{5}=T_{3}-T_{4}+\frac{T_{a b}}{2}=\left(T_{1}-T_{2}\right) \times \frac{\left(E_{3}+E_{4}+E_{i 1}+E_{5}\right)}{E_{i 1}+E_{3}}$.

The AC signal loaded on the voltage at both ends to be measured passes through the amplifying circuit; the AC signal is amplified, but the DC signal remains unchanged. The multiple $\mathrm{N}$ of AC signal amplification is expressed as

$$
N=\frac{D_{x}}{D_{x}+2 E} \times \frac{\left(E_{3}+E_{4}+E_{i 1}+E_{5}\right)}{E_{i 1}+E_{3}} .
$$


It can be seen from equation (8) that when $E$ is fixed, the magnification factor is only related to the resistance of $D_{x}$, and the greater the $D_{x}$, the greater the magnification $N$.

\subsection{Sine Fitting Algorithm and Difference Algorithm}

2.3.1. Difference Algorithm. The algorithm is the inverse operation of the prefix sum algorithm, which can quickly calculate a certain interval of the array. The difference algorithm adopted is to subtract the second half-period data of the four adjacent half-periods from the first half-period data correspondingly [29]. After each difference, the amplitude of the data will increase by 2 times, and the data cycle will lose the data of the last half cycle. In this paper, the sampling frequency of the output signal is $1 \mathrm{kHz}, 120$ data points are sampled in one cycle, and the sampling frequency is 120 times the fundamental frequency. The frequency of noise eliminated by this system is $50 \mathrm{~Hz}$, so the period is exactly 20 times the effective signal. Among them, $n(x)$ is the original sampled data, and $m(x)$ is the data after differential processing.

The difference formula is

$$
\begin{aligned}
n(x)= & m(x)-4 m(x+20)+6 m(x+50) \\
& -4 m(x+80)+m(x+110) .
\end{aligned}
$$

2.3.2. Sine Fitting Algorithm. When the output signal is not saturated, this article adopts a digital fitting method of amplitude and phase. It adopts the method of summing first, then multiplication, which saves processing time and improves measurement efficiency $[4,30]$. Assuming that the number of sampling cycles is $n$ and the amount of sampled data in each cycle is $N$, only $(n-1) N$ addition operations and $N$ multiplication operations are required, and the result of the sine function is solidified in the memory, which saves time. In the fitting process, for a sine signal with a periodic frequency of $1000 \mathrm{~Hz}$ and a quantity of 120, full use of the symmetry of the sine and cosine signals can form a complete full-period data, saving storage space. It inputs the sine function into the previously trained model, and the sine function is used for training to improve versatility.

The sine fitting solution formula can be simplified as

$$
\left\{G_{i}=\sum_{i=0}^{x} x_{i}(a) y_{i}(b) K_{i}=\sum_{i=0}^{x} x_{i}(a) z_{i}(b)\right.
$$

Then, the amplitude and phase can be calculated by formula or function, respectively:

$$
\left\{\begin{array}{l}
k_{i}=\sqrt{x_{i}^{2}+y_{i}^{2}} \\
\mu_{i}=z \sin 2\left(-z_{i}, x_{i}\right)
\end{array}\right.
$$

The characteristic of complex impedance is that when a certain signal is input, there is not only a difference in amplitude between the output amplified signal and the input signal, but also a certain phase difference. Therefore, the measurement of complex impedance is essential in obtaining the amplitude ratio and phase difference between the output signal and the input signal. It uses the result of synchronous acquisition to obtain the amplitude and initial phase of the input and output signals from the perspective of fitting, thereby obtaining the amplitude and phase difference ratio.

The calculation can be expressed as

$$
w_{i}=X \cos \left(\varepsilon+\kappa_{i}\right)+\varepsilon_{i}+Z .
$$

Expanding the formula to

$$
w_{i}=X \cos \varepsilon_{i}+Y \sin \varepsilon_{i}+Z+\varepsilon_{i} .
$$

Using the least squares fitting formula, set an error objective function:

$$
g(x, y, z)_{\min }=\sum \kappa_{i}^{2}=\sum\left[w_{i}-\left(x \cos \kappa_{i}+y \sin \kappa_{i}+z\right)\right] .
$$

To minimize parameter $\kappa$, the partial derivatives of variables $a, b$, and $c$ need to be zero, satisfying

$$
\begin{aligned}
& \frac{\sigma g}{\sigma x}=2 \sum \cos \kappa_{i}\left[w_{i}-\left(x \cos \kappa_{i}+y \sin \kappa_{i}+z\right)\right]=0, \\
& \frac{\sigma g}{\sigma y}=2 \sum \sin \kappa_{i}\left[w_{i}-\left(x \cos \kappa_{i}+y \sin \kappa_{i}+z\right)\right]=0, \\
& \frac{\sigma g}{\sigma z}=2 \sum\left[w_{i}-\left(x \cos \kappa_{i}+y \sin \kappa_{i}+z\right)\right]=0 .
\end{aligned}
$$

When the output signal is saturated, the method of not changing the sine signal of muscle strength and the amplification factor of the amplifier is adopted, and the saturated data is excluded from the calculation sequence. The least square method is used to calculate the saturated sinusoidal signal and obtain the signal amplitude and phase parameters. After sorting, we can get

$$
\begin{aligned}
\sum w i \times \cos \kappa_{i} & =x \times \sum \cos ^{2} \kappa_{i}+y \times \sum \sin \kappa_{i} \cos \kappa_{i}+z \times \sum \cos \kappa_{i}, \\
\sum w i \times \sin \kappa_{i} & =x \times \sum \sin \kappa_{i} \cos \kappa_{i}+y \times \sum \sin ^{2} \kappa_{i}+z \times \sum \sin \kappa_{i}, \\
\sum w i & =x \times \sum \cos \kappa_{i}+y \times \sum \sin \kappa_{i}+z \times \sum 1 .
\end{aligned}
$$


Finally, the fitted sine signal is compared with the input signal to obtain the amplitude ratio and phase difference. And substitute the amplitude ratio into the resistance value to be measured, and judge the speed according to the curve of resistance value change with time.

\section{The Experimental Design of Wing Chun Day Punch Boxing Based on Embedded Microprocessor}

3.1. Experiment Object. Kinematics is mainly to study the position changes of objects in the spatial structure, through the system to measure the time nodes and spatial changes of the object's movement, mark the start time, end time, and duration of the movement, and quantify the gaps in the frequency and rhythm of the movement. Kinematics mostly takes human movement or the movement of other animals as the research object, focusing on analyzing the displacement, velocity, acceleration, and force of different body joints, center of gravity, and force points through statistics and calculations. Biomechanics is the branch of biophysics that applies the principles and methods of mechanics to the quantitative study of mechanical problems in organisms. Myoelectricity is a phenomenon in which nerves and muscles are stimulated to cause excitement and generate bioelectricity. In sports science research, electromyography is mainly used for the diagnosis of muscle strength and muscle fatigue and can also be used to assess the training level of athletes. In low-level athletes, the myoelectric activity is disorderly, and the excess muscles participate in the discharge. And athletes with higher training levels have regular muscle discharges, showing a certain regularity, and there will be no excess muscle discharges. This study uses electromyography to evaluate the sequence of muscle activation and the sequence of muscle exertion during Wing Chun Japanese punching, as well as the coordination relationship between the muscles and the sequence of stopping activity. It can find out the characteristics and mechanism of Wing Chun's force release through the law of the discharge of each force muscle.

This subject research is aimed at the analysis of the effect of Wing Chun Japanese punch boxing and does not involve influencing factors to judge the "quality" of the pros and cons of the action. Due to the fact that it is difficult for highlevel Wing Chun subjects to find it, four Wing Chun practitioners are selected as the research objects, who have rich martial arts experience and age in Wing Chun. The specific information is shown in Table 1 .

\subsection{Experiment Preparation}

3.2.1. Preparation before the Experiment. All subjects voluntarily participated in the test of this item and did not do vigorous exercise within 24 hours before the test, in order to eliminate the interference of muscle fatigue factors. And inform the subjects in advance of the specific procedure of the biomechanics experiment of the whole Wing Chun leaning hammer movement. The experimental staff installed and tested the force plate, high-speed camera, and electromyography equipment to ensure the smooth progress of the test. The test environment has the same temperature, and the subjects fully warmed up to reduce the viscosity of their muscles and prevent the testers from being injured.

3.3. Experimental Parameter Setting. The tested engineering dummy is equipped with a simulated visceral tissue filler inside the thorax, and a force or acceleration sensor is installed in important visceral parts, which can simulate the stress of the victim's internal relevant parts and the displacement acceleration of the hit part. The joint angles selected for the subject research are as follows: left hip angle, left knee angle, left shoulder chest angle, left elbow angle, left hip angle, right knee angle, clamp male angle, eccentric horse angle, and left shoulder angle. For example, it is used to test whether the principle of "keeping the middle and using the middle" is followed when using the partial body hammer punching punch; Clamp Yang Horse Horn is used to test the two-character box Yang Horse of Wing Chun Boxing, and the partial horse horn is used to test Wing Chun's Lean Horse, test muscles, and collect angle and displacement information. Figure 4 shows the experimental frame of the embedded microprocessor on the impact analysis of Japanese punch boxing.

The processing of Wing Chun Japanese Chongquan is mainly divided into two steps: one is to preprocess the data, mainly for the stage of collecting data. Due to some uncontrollable circumstances, the collected data curve is not coherent, and the preprocessing is to complete the vacant data. In the completion process, it is necessary to ensure that the inserted data curve is continuous and accessible, because the angular velocity, angular displacement, and acceleration curves of the moving object must be determined and calculated through the curve to ensure the continuity of all data curves. The second is to perform comprehensive calculations on the motion. In the process of calculation, each department of the moving object is considered as a relatively rigid body for research. For example, the movement of the right shoulder joint, elbow joint, and wrist joint in the Wing Chun Day punch can be calculated by the principles of physical mechanics and other principles of the joint force and force transmission. At the same time, the rigid body adhering to the human body will also move at any time to form the same motion state as the human body. It describes the motion state of these cylinders through the coordinate system, and then some data such as the displacement, velocity, angular velocity, and acceleration of the joint points of the human body can be obtained.

3.4. Experimental Method. This experiment uses Simi Motion analysis system to analyze motion technology, acquires images through two high-speed cameras, and performs 3D motion image analysis. In the analysis, the marker points of each part are first determined, and then the videos taken by two high-speed cameras are imported, the action videos to be analyzed are selected, and the three-dimensional frame parameters are determined. After the preparatory work is 
TABLE 1: Basic situation of experimental subjects.

\begin{tabular}{lccccc}
\hline Serial number & Height $(\mathrm{cm})$ & Weight $(\mathrm{kg})$ & Age & Boxing age & Sport level \\
\hline A & 176 & 69 & 26 & 6 & Level 2 \\
B & 181 & 74 & 28 & 5 & Level 2 \\
C & 172 & 68 & 25 & Level 2 \\
D & 168 & 70 & 29 & 10 & Level 2 \\
\hline
\end{tabular}

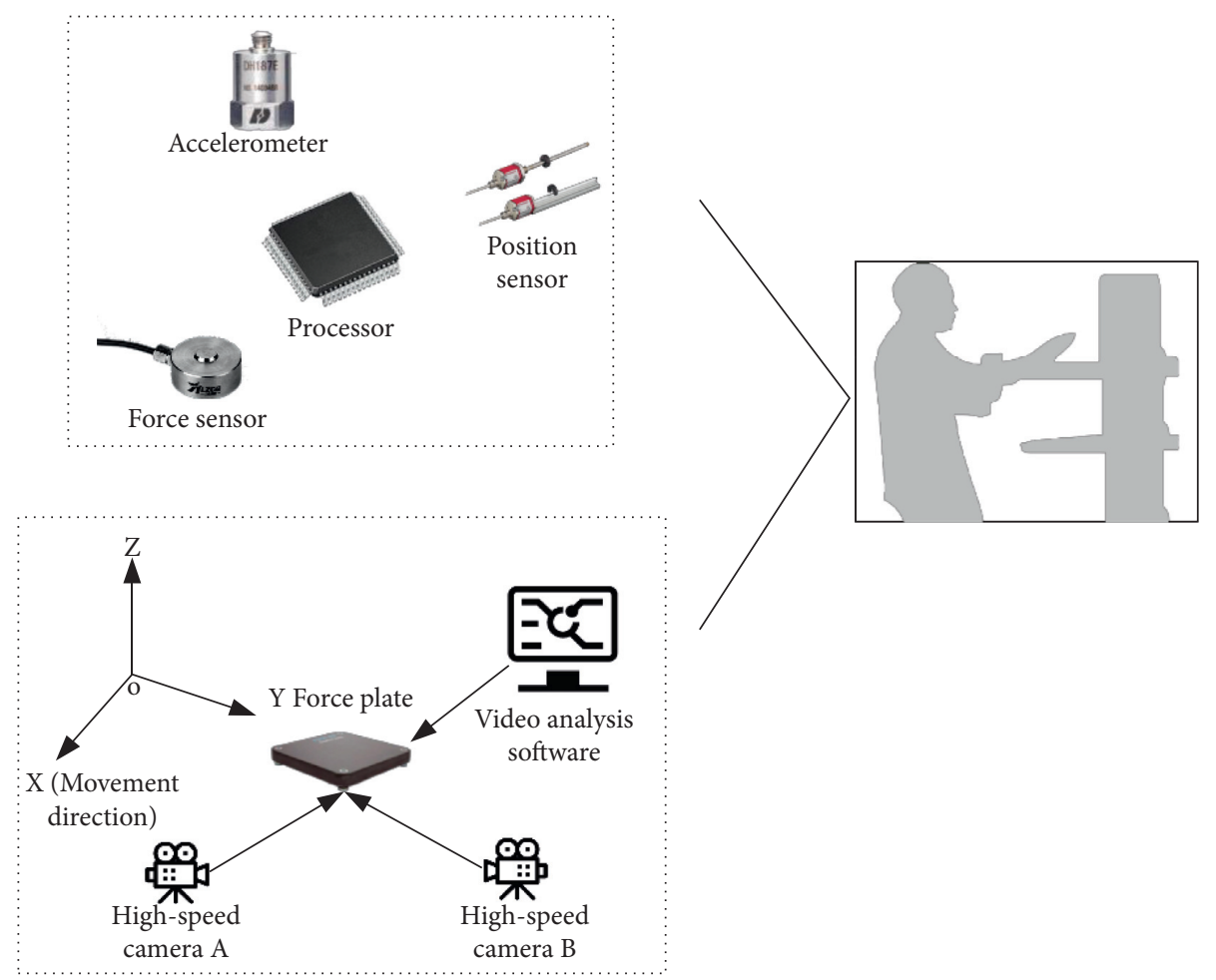

FIGURE 4: Experimental frame diagram of the analysis of the impact of the embedded microprocessor on the Japanese punch boxing.

completed, the 3D images formed by the two high-speed cameras are traced, and three-dimensional analysis is performed, and finally the motion data of various parts of the human body is calculated.

The subjects affixed reflective marker balls in accordance with the unified standard, unified the test-specific clothing, and used the MX_F40 camera to jointly capture the kinematics data of the subjects during the test action. The Swiss Kistler force plate is used to test the ground reaction force data generated by the force of the feet during the action process. In this experiment, the sports biomechanics of Wing Chun Japanese punching boxing was analyzed, and the electromyography of the surface muscles was collected using an electromyography meter. The muscles selected in the test are all on the right side: lateral head of calf triceps, right rectus femoris quadriceps, pectoralis major, anterior deltoid, triceps, biceps, and brachioradialis. The electromyography test system is used, which tests the biological signals of different phases and intensity changes of muscle work when the movement is completed and understands the working condition of the muscles during the movement. Three-dimensional kinematics tests the angle and angular velocity transformation of each joint of the subject's body; the force plate obtains the synchronized change process of the angle and magnitude of the ground force; the wireless surface electromyography instrument synchronously records the muscle activation sequence and duration data during the whole process.

After the preparatory work is completed, the subjects are familiar with the entire process, and then they step on the force plate with both feet following the instructions of the experimenter. They stood up in the posture of Wing Chun, tongs, and horses and began to punch Japanese punches, while the experimenters turned on the experimental equipment to simultaneously collect experimental data. The force plate, EMG, and high-speed photography must be synchronized during data collection. If one of the parties is not synchronized, the experiment will be invalid, and try again. Each subject does "Japanese punch punches" in place, and each group punches three times in a row and collects data on the actions completed by each subject three times, confirms that they are correct, saves them, and replaces them with another person. After the synchronization experiment is over, the test method is changed to allow different subjects to use the same technical movements, such as the right and left straight fist combo style, with one left and one right as a 
set of movements. In the combo test, the subject must complete the combo continuously within 30 seconds, starting with the actual combat posture for each movement. After performing a set of actions, return to the actual combat position again, perform the next combo action, and repeat this for 30 seconds. Wing Chun uses two-character forceps Yangma to strengthen the stability of the lower limbs, maintain body balance, and use the ground reaction force and the changes in the joint angles of the whole body to develop the overall strength. Through the analysis of the joint angles, the action characteristics of Japanese punching boxing are obtained. Then, it conducts isokinetic tests on the subjects' wrist joints, hip joints, shoulder joints, elbow joints, etc. respectively. According to the time difference between the professional group and the general group at the same speed, the key factors affecting the right straight punch are analyzed. Each subject is required to fully adjust the physical strength and state, perform a normal level in each action, and finally count all the subject data of each subject's hitting power and total number of hits per unit time. After the successful acquisition is completed, save it, clean up the experimental site, and recover the instrument.

\section{The Experimental Analysis of the Effect of Wing Chun Japanese Punching Boxing Based on Embedded Microprocessor}

\subsection{Japanese Punching Movements}

4.1.1. Perspective Analysis. It organizes the collected kinematics and dynamics data in the software, inputs static theme files to create a model, reads theme-based theme animation files, and completes model assignment tasks. It then performs a series of calculations on the selected coordinate system, single link, and reference link for hip, knee, elbow joint angles, and punch point speed. Figures 5 and 6 are the statistical results of the subject's left and right knee angles and hip angles.

From the above statistical analysis, it can be known that the external torque of these joints received by the subjects during the Japanese punch does not change much, which is not conducive to the rotation of the joints. And it is used to maintain the balance and stability of the body, which shows that strengthening the stability of the lower limbs can effectively maintain the balance of the body. The subjects as a whole showed significant changes in the left and right knee joints and right hip joints, indicating that the subjects used their waist strength when doing Japanese punches. The waist is known as the center of strength in martial arts, and proper waist movements are conducive to the transfer of moments of momentum in various parts of the body. Although the waist was used at the same time, the comparison of the mean values of Subject B and Subject C with the other two subjects found that the right hip angle of Subject B changed more significantly than that of the other subjects, and it shows that the perennial Wing Chun practitioners are better than beginners in using the waist. Although beginners use the waist, they are not good at using the changes in the male horns of the forceps and the joint angles of the left and right knees to promote the rotation of the moment of momentum. One of the reasons why Wing Chun masters exert their amazing power is to make full use of the changes in the angle of each joint to promote the transmission of momentum.

Figure 7 shows the changes of the knee joint angle on the punch side and the contralateral side, respectively. It can be seen from Figure 7 that the angle of the knee joint on the punch side of the subject changed about $10^{\circ}$, and the angle of the contralateral knee joint did not exceed this range. Among them, the change of the knee joint angle of Subject A was the smallest, the change angle of the punch side was $6^{\circ}$, and the contralateral side was only $2^{\circ}$, and such a small change would be easily ignored by naked eye observation.

Figure 8 is the statistical data of the subject's left and right shoulder and chest angles.

Wing Chun requires the elbow and the body to keep a punch distance and remember that the arm is close to the body. The reason for this is that one is to increase the distance between the forearms; the second is to maintain a proper angle of the elbow joint to facilitate punching; third, it is good for defense. People who have practiced Wing Chun often have this experience when their arms are close to the body; they are easily blocked with their hands. The peaks of the angular velocities of the knee joints, hip joints, and shoulder joints appear very close in time. The maximum angular velocity of the knee joint appears first, and then the peaks of the angular velocity of the hip joint and the angular velocity of the shoulder joint appear almost simultaneously, but the angular velocity of the knee joint is significantly higher than the angular velocity of the shoulder joint. It can be seen that the movement of the flat upper part on the left is mainly guided by the movement of the small joints by the large joints. To reach the maximum speed, the subject's shoulders and upper limbs must move as hard as possible in the attacking direction to complete and adjust the direction of movement of the Japanese punch. Therefore, the coordinated training of the body's joints is an effective means to improve the hitting effect.

Figures 9 and 10 are descriptive statistics of the angles of the left and right elbow joints in Japanese punching. The toggle joint is in the "two pass" of Wing Chun, that is, between the wrist joint and the shoulder joint. In Guangzhou Wing Chun twelve styles, the striker can suppress the opponent's elbow and attack the opponent's midline at the same time. The elbow joint has the famous "Principle of Unmoving the Elbow" in Wing Chun. At the same time, it emphasizes the return of the elbow, which means that the elbow joint prevents the opponent from grabbing the center line when attacking or defending the center line, which is beneficial to protect the important organs in the two ribs. Therefore, the angle of the elbow joint is extremely important in Wing Chun, and a good joint angle makes the punch faster and stronger. The angle of the elbow joint of the Japanese punch is the most obvious, and the tendency of the angle of the elbow joint of the four subjects is almost the same. About $70 \%$ to $80 \%$ of its punches drop to the minimum and then quickly rise to the maximum angle (about $160^{\circ}$ ). In general, the angle of the elbow joint changes before $70 \%$ of the Japanese punches and then enters a rapid 

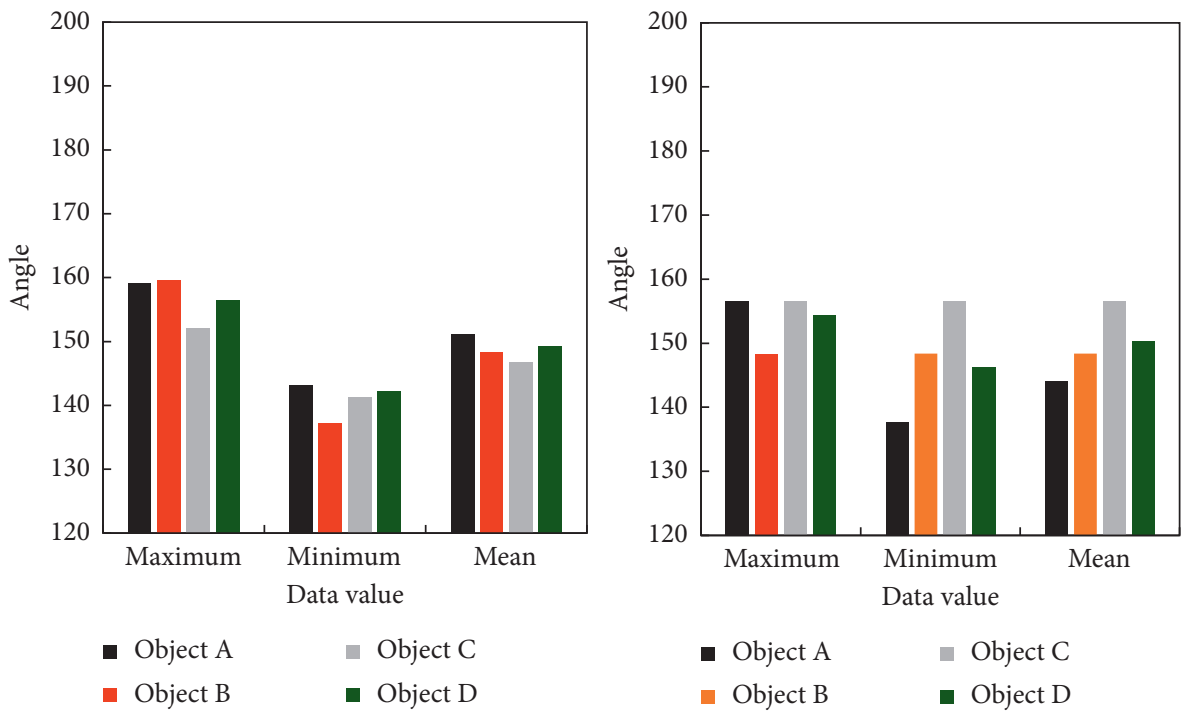

Figure 5: Statistics of subjects' left and right knee angles.
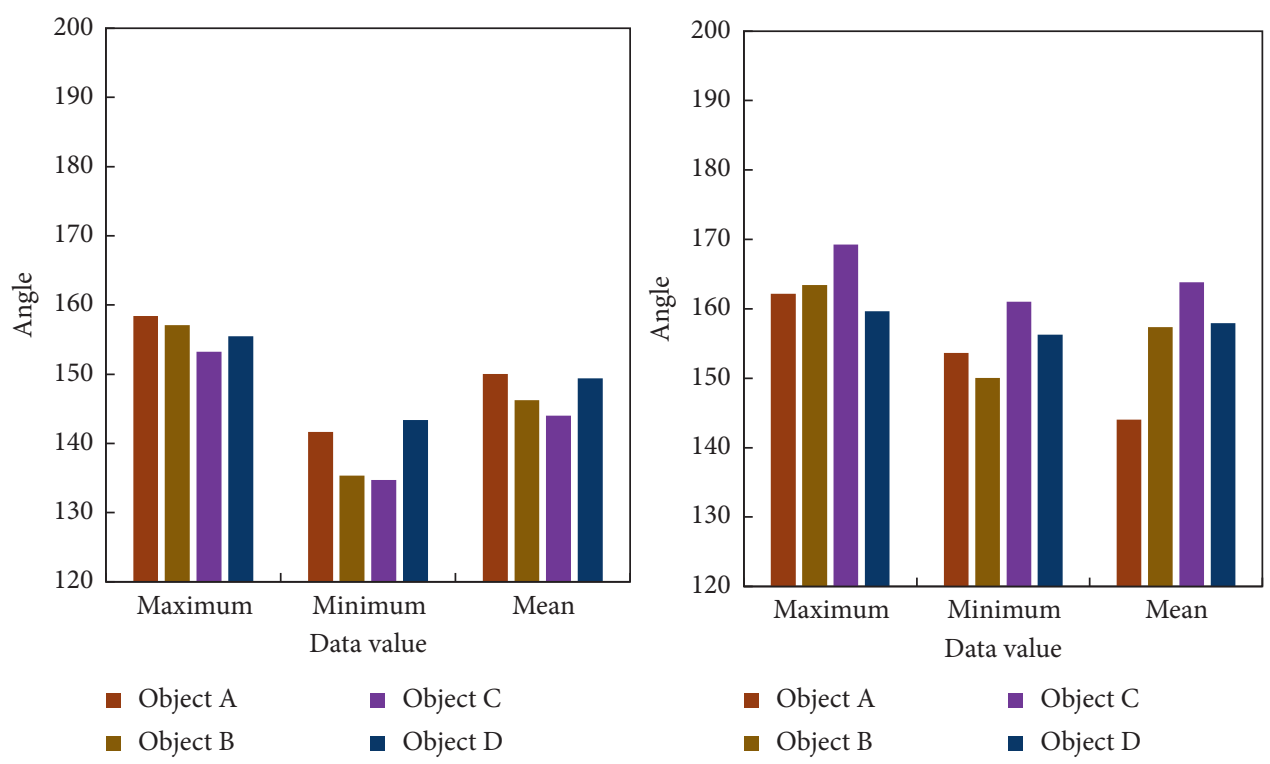

FIGURE 6: Statistics of subjects' left and right hip joint angles.

change phase. It can be seen that, in Japanese punching fist, the elbow joint basically maintains the initial angle, and the back elbow joint is quickly straightened forward until the movement is completed.

Through analysis, it can be found that the shoulder, elbow, and wrist joints reach the peak speed in different phases with the extension of the action. Among them, the speed curve of the shoulder joint is the first to change, and it is the first to reach the peak speed of the three joints. The speed curves of the elbow joint and the wrist joint have similar changes, and both are relatively stable in the early stage, and then the speed suddenly increases and finally drops suddenly, with a large peak speed. The speed changes of the shoulder, elbow, and wrist joints indicate that, in the right straight punch technique, the shoulder joint drives the movement of the elbow and wrist joints; that is, the small joints are driven by the large joints. This is consistent with the human body's motion conditions, the large joints are closer to the axis of the human body, and the small joints are farther from the axis of the human body. Therefore, when the human body moves, the proximal joints first move and shift and then drive the movement and force of the distant joints.

4.1.2. Analysis of Punching Speed. Speed is the key to your boxing technique, and only when punching quickly can the opponents be overwhelmed by defense. In particular, Wing Chun, which pays attention to close combat, needs to punch quickly and powerfully within a limited time and within a 

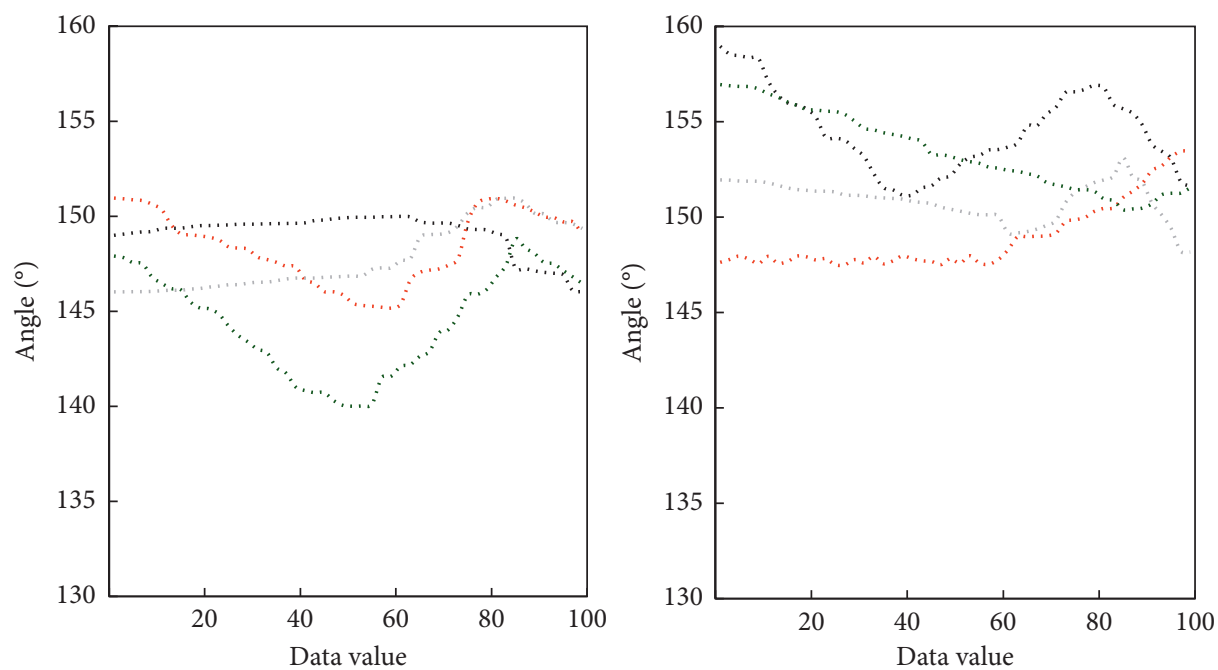

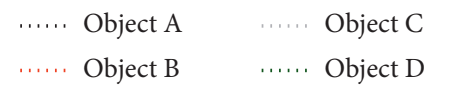

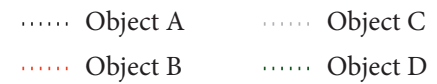

FIgURE 7: Changes in the knee joint angle on the punch side and the opposite side.

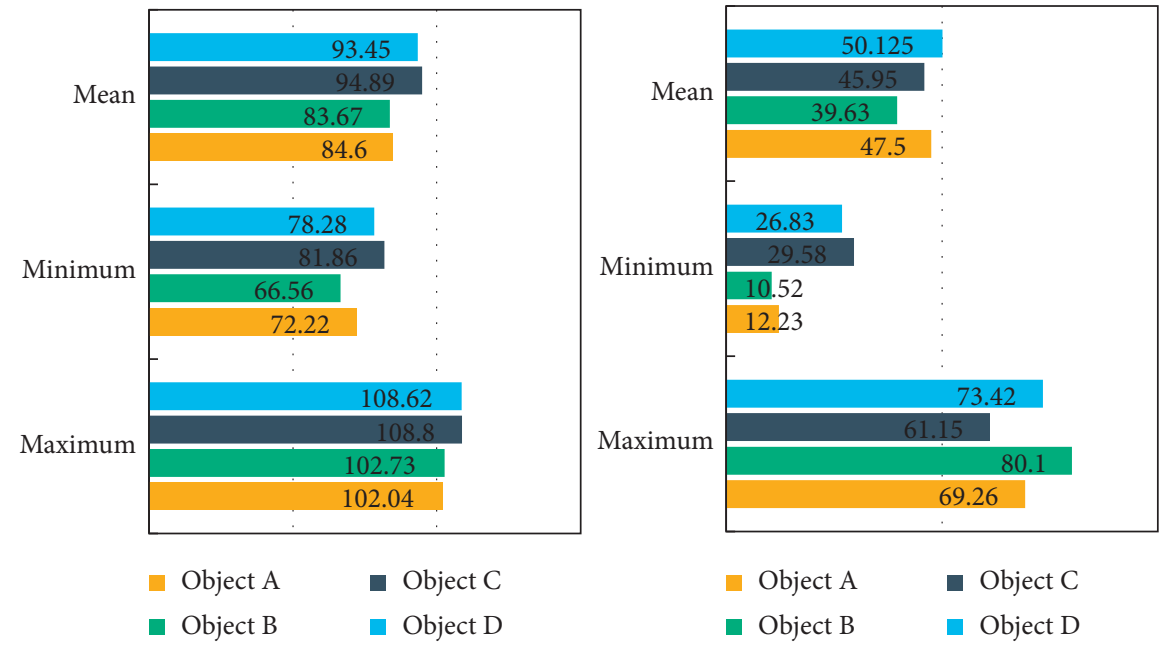

Figure 8: Statistics of the subjects' left and right shoulders and chest angles.

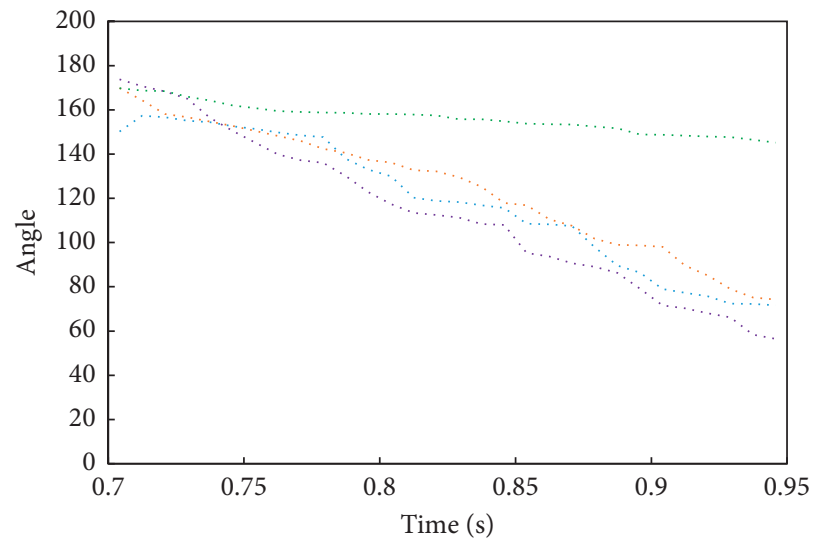

Object A

Object C

Object B

Object D

FIGURE 9: Descriptive statistics of right elbow joint angle. 


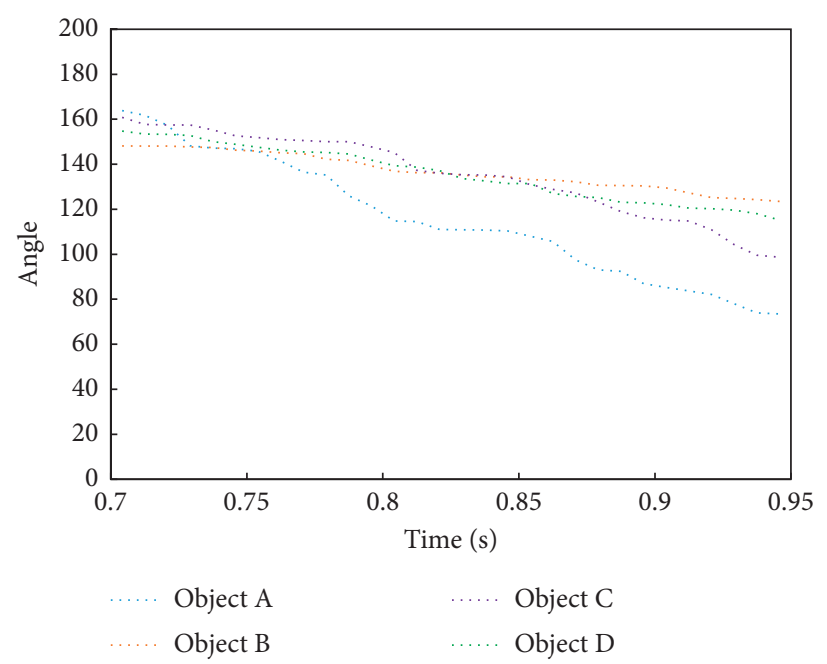

Figure 10: Descriptive statistics of left elbow joint angle.

very short distance in order to strike the opponent with lightning speed and win the fight. The subject's punching speed was analyzed, and the detailed data of the subject's left and right punching speed are shown in Tables 2 and 3.

As shown in Tables 2 and 3, when the four subjects performed the Japanese punching movement, the left and right punches were performed at the same time, the left punch was punched out, the right punch was closed, and the right punch was punched out, and the left punch was closed, and among them, the speed of the right fist of the subjects was generally faster than that of the left fist. After they pass the back hand, they draw their punches to exert force, and the power is transmitted through the back to the left waist and hips, and then from the waist and hips to the fist surface. The longer the route of power transmission, the greater the momentum conversion, and the greater the hitting effect, which reflects the overall strength of Wing Chun.

4.2. The Effect of Japanese Punching Boxing. Power tests have appeared in various sports and medical research fields, and martial arts, as a sport that pursues martial arts effects and performance, attach great importance to striking power. Engineering dummies are highly bionic dummies that can effectively study the damage caused by collisions. This article uses engineering dummy for experiments and analyzes the hitting effect of Japanese punching boxing by collecting relevant data of engineering dummy. The most important thing is to record and compare the acceleration of the "sternum" and "heart" of the engineering dummy when it is hit. Through the comparison, the actual effect of the hit can be judged, and then the most reasonable punch angle, muscle work, and joint movement characteristics of the right-hand back straight punch technique can be judged. Acceleration sensors are the most important and commonly used data collection points in engineering dummies. It is located inside the breastbone, just in front of the stainless steel breast structure of the engineering dummy, and is close to the surface of the engineering dummy, simulating the muscular skin. The subject's impact force acts on the mechanic through the skin layer of the superficial muscles, causing accelerated movement and detailed numerical changes throughout the process. The data measured by the acceleration sensor may largely reflect the changes in the related indicators of the mechanics test due to the frontal impact. Figure 11 is the peak acceleration of different striking actions.

The maximum accelerometer value of the visceral shock caused by various actions was input into the SPSS software for statistics, and it was found that the average number of acceleration peaks of the sternal shaft in each group was relatively close. However, there was no difference in the $T$ test between the two groups, and at a normal distance, it can be seen that there is no statistically significant difference between the frontal impact of the Japanese punching fist shoulder and the engineering dummy test item and the resulting peak ground acceleration. After SPSS software $T$ test analysis, an engineering dummy hits the prepared $150^{\circ}$ elbow joint to record the changes in the peak data of the sternum accelerometer, and the average number of accelerometers in the two groups is different.

Table 4 is an analysis of the difference of the oscillating acceleration values of internal organs. It can be seen from the table that the calculated values are all smaller than the rejection range. It is believed that there is no significant difference in the results of the visceral shock acceleration value applied to the engineering dummy by different actions of the subjects, and the results of the subjects' Japanese punching punches are relatively stable. In the case of $150^{\circ}$ elbow preparation, the midline punch was significantly larger than the punch directly in front of the shoulder, and the difference was highly statistically significant (significant level $p<0.01$ ). Analysis of the vibration acceleration of the internal organs shows that the normal punch distance not only is larger than the short distance punch in the impact force peak caused by the impact surface, but also causes greater damage to the opponent's internal organs. 
TABLE 2: Descriptive statistics of left fist speed of subjects.

\begin{tabular}{lcccc}
\hline Subject & Maximum value & Minimum value & Average value & Standard deviation \\
\hline A & 4.148 & 0.142 & 1.316 & 0.706 \\
B & 3.976 & 0.158 & 1.284 & 0.642 \\
C & 3.845 & 0.204 & 1.262 & 0.847 \\
D & 3.543 & 0.283 & 1.189 & 1.523 \\
\hline
\end{tabular}

TABle 3: Descriptive statistics of right fist speed of subjects.

\begin{tabular}{lcccc}
\hline Subject & Maximum value & Minimum value & Average value & Standard deviation \\
\hline A & 6.425 & 1.421 & 1.544 & 1.572 \\
B & 4.761 & 0.183 & 1.206 & 0.638 \\
C & 4.356 & 0.245 & 1.227 & 0.715 \\
D & 4.482 & 0.276 & 1.214 & 1.741 \\
\hline
\end{tabular}

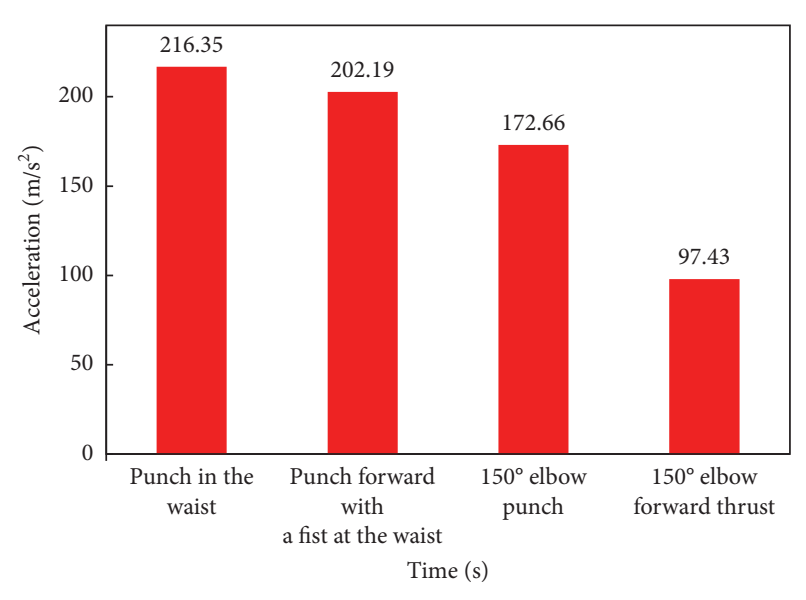

FIGURE 11: Peak acceleration of different punching movements.

TABLE 4: Analysis on the difference of visceral acceleration value of visceral organs.

\begin{tabular}{lccccc}
\hline Organ & A & B & C & D & T \\
\hline Heart & 2.1809 & 1.5662 & 1.3935 & 3.9922 & \\
Liver & 3.3292 & 0.4482 & 1.2366 & 0.6743 & \\
Left lung & 0.4710 & 2.1806 & 0.6543 & 2.5004 & $<0.01$ \\
Right lung & 1.3287 & 2.0341 & 1.1026 & 1.3082 & \\
\hline
\end{tabular}

\section{Conclusions}

Traditional martial arts call for more research that highlights the practical significance. Through the research and analysis of the striking effect of Wing Chun Japanese Punch Boxing, the actual effect of Wing Chun Boxing can be displayed more clearly, and at the same time, a deeper martial arts culture can be revealed. This article is based on the embedded microprocessor to study the punching effect of Wing Chun Japanese punch, using force plate and engineering dummy to analyze the blow effect of the subjects. It reflects and simulates the stress of the internal part of the victim and the displacement acceleration of the affected part and analyzes the vibration acceleration value of the internal organs of the engineering dummy under different actions to obtain the actual hitting effect. It can be seen from the results of the research in the article that the different force movements of Japanese punching boxing will not have a significant difference in the striking effect. There are still some shortcomings in this article. For example, this experiment uses high-speed video, which is limited by equipment and cannot be analyzed from some angles. It is hoped that if conditions permit, a more advanced three-dimensional analysis system should be used to carry out a deeper analysis of the Wing Chun Japanese Punch Boxing.

\section{Data Availability}

No data were used to support this study.

\section{Conflicts of Interest}

The authors declare that there are no conflicts of interest with any financial organizations regarding the material reported in this manuscript.

\section{References}

[1] P. Perkins, A. Jamieson, and W. Spratford, "Effect of pneumatic boxing gloves on impact kinematics and their relationship to impact forces," World Journal of Engineering and Technology, vol. 7, no. 3, pp. 472-512, 2019.

[2] S. Kasiri, C. Fookes, S. Sridharan, and S. Morgan, "Finegrained action recognition of boxing punches from depth imagery," Computer Vision and Image Understanding, vol. 159, pp. 143-153, 2017.

[3] Y.-B. Lee, D.-T. Lee, B.-Y. Hwang, Y.-S. Son, and J.-G. Kim, "Energy expenditure changes of individual boxing movement following training," The Korean Journal of Physical Education, vol. 56, no. 3, pp. 539-549, 2017.

[4] M. Filipe and Lins, "Register file criticality and compiler optimization effects on embedded microprocessor reliability," IEEE Transactions on Nuclear Science, vol. 64, no. 8, pp. 2179-2187, 2017.

[5] R. Sato, Y. Hatanaka, Y. Ando et al., "High-speed operation of random-access-memory-embedded microprocessor with minimal instruction set architecture based on rapid single- 
flux-quantum logic," IEEE Transactions on Applied Superconductivity, vol. 27, no. 4, pp. 1-5, 2017.

[6] M. A. Boussadi, T. Tixier, A. Landrault, and J.-P. Derutin, "HNCP: a many-core microprocessor ASIC approach dedicated to embedded image processing applications," Microprocessors and Microsystems, vol. 47, pp. 333-346, 2016.

[7] L. T. Clark, D. W. Patterson, C. Ramamurthy, and K. E. Holbert, "An embedded microprocessor radiation hardened by microarchitecture and circuits," IEEE Transactions on Computers, vol. 65, no. 2, pp. 382-395, 2016.

[8] P. W. Davis, R. Waldock, A. Connorton, S. Driver, and S. Anderson, "Comparison of amateur boxing before and after the 2013 rules change and the impact on boxers' safety," British Journal of Sports Medicine, vol. 52, no. 11, pp. 741-746, 2018.

[9] M. Urrutia, C. Ivy, P. S. Pohl, and L. Denney, "Boxing to improve sleep quality and daytime sleepiness in individuals with Parkinson disease," Topics in Geriatric Rehabilitation, vol. 36, no. 3, pp. 170-175, 2020.

[10] M. Lefik and Z. Gmyrek, "Numerical modelling of a fluxbarrier SynchRel motor including punching effect," COMPEL International Journal of Computations and Mathematics in Electrical, vol. 35, no. 6, pp. 2022-2034, 2016.

[11] L. Xin, G. Jia, and M. Zhou, "RNA binding protein QKI contributes to WT1 mRNA and suppresses apoptosis in ST cells," Genes \& Genomics, vol. 39, no. 9, pp. 1-8, 2017.

[12] Y. Sakino, K. Yoshikawa, Y. Sano, and R. Sumiya, "Effect of laser peening on improving fatigue strength of welded rib of high-strength steel," Quarterly Journal of the Japan Welding Society, vol. 34, no. 1, pp. 20-25, 2016.

[13] P. Dallmann, C. Bach, H. Zipser, P. A. Thomann, and S. C. Herpertz, "Evaluation of a stress prevention program for young high-performance athletes," Mental Health \& Prevention, vol. 4, no. 2, pp. 75-80, 2016.

[14] J. Xing, F. Wang, Q. Xu et al., "Screening of potential probiotic lactic acid bacteria based on gastrointestinal properties and perfluorooctanoate toxicity," Applied Microbiology and Biotechnology, vol. 100, no. 15, pp. 6755-6766, 2016.

[15] L. Desbois, P. Tchoreloff, and V. Mazel, "Influence of the punch speed on the die wall/powder kinematic friction during tableting," Journal of Pharmaceutical Sciences, vol. 108, no. 10, pp. 3359-3365, 2019.

[16] Daiki and H. Ichikawa, "Effect of needle punching process on a chopped strand mat composite with an open hole," Open Journal of Composite Materials, vol. 8, no. 3, pp. 110-123, 2018.

[17] O. I. Khalaf and G. M. Abdulsahib, "Energy efficient routing and reliable data transmission protocol in WSN," International Journal of Advances in Soft Computing and Its Applications, vol. 12, no. 3, pp. 45-53, 2020.

[18] Z. Lv, R. Lou, and J. Li, "Big data analytics for 6G-enabled massive internet of things," IEEE Internet of Things Journal, vol. 8, no. 7, pp. 5350-5359, 2021.

[19] Y. Zeng, G. Chen, K. Li, Y. Zhou, X. Zhou, and K. Li, "Mskyline: taking sunk cost and alternative recommendation in consideration for skyline query on uncertain data," Knowledge-Based Systems, vol. 163, no. 1, pp. 204-213, 2019.

[20] M. Katoh and T. Aihara, "Effect of mass of drop-hammer in impact punching with PVA gel as working medium," Japan Society for Technology of Plasticity, vol. 60, no. 697, pp. 27-32, 2019.

[21] A. Ibrahim, S. E. E. El-Metwally, H. H. Asker, and M. A. El Zareef, "Effect of mid-thickness rebar mesh on the behavior and punching shear strength of interior slab-column connection," HBRC Journal, vol. 12, no. 3, pp. 272-283, 2016.
[22] C. Gong, J. Liang, and G. Wen, “An image measurement method of whole field deformation during thin plate welding process," Journal of Central South University (Science and Technology), vol. 48, no. 11, pp. 2935-2941, 2017.

[23] S. Nagasawa and S. Ozawa, "Effect of bending velocity on time-dependent release behavior of creased white-coated paperboard," Mechanical Engineering Journal, vol. 3, no. 3, pp. 16-182, 2016.

[24] H. Chen and Y. Sun, "Development and application of reliability test platform for high-speed punch machine clutch brake system," Journal of Mechanical Science and Technology, vol. 31, no. 1, pp. 53-61, 2017.

[25] M. L. Liu and X. L. Chen, "Clinical characteristics and therapeutic effect of open globe injury," International Eye Science, vol. 16, no. 6, pp. 1199-1202, 2016.

[26] K. Reals, "Boxing clever," Airline Cargo Management, vol. 15, no. 1, pp. 16-18, 2016.

[27] C. Ckay and K. Stokes, "The crowd goes wild: the dissemination of concussion risk information on twitter in response to the removal of headgear in men's boxing at the olympic games," British Journal of Sports Medicine, vol. 51, no. 4, pp. 361-363, 2017.

[28] R. Schule, "A comparison of bone mineral density in amateur male boxers and active non-boxers," International Journal of Sports Medicine, vol. 37, no. 09, pp. 694-699, 2016.

[29] M. Kociuba, S. Kozie, and R. Chakraborty, "Sports preference and digit ratio $(2 \mathrm{~d}: 4 \mathrm{~d})$ among female students in Wrocaw, Poland," Journal of Biosocial Science, vol. 49, no. 5, pp. 1-11, 2016.

[30] U. von Hecker, U. Hahn, and J. Rollings, "Spatial representation of coherence," Journal of Experimental Psychology: General, vol. 145, no. 7, pp. 853-871, 2016. 